Women and Children's Health, San Bortolo Hospital of Vicenza, Vicenza, Italy; ${ }^{4}$ Department of Transfusion Medicine, San Bortolo Hospital of Vicenza, Italy, Vicenza, Italy; ${ }^{5}$ University of Padua, Department of Medicine-DIMED, Padova Italy; ${ }^{6}$ San Bortolo Hospital Of Vicenza, Department of Medicine, Vicenza, Italy

Background: Therapeutic apheresis (TA) represents a therapeutic option in pre-existing conditions or rheumatic diseases that occur during gestation. Although pregnancy is not a contraindication itself, due to the lack of evidence-based guidelines and the alleged risk of maternal and/or fetal adverse events, there is general resistance to its application during pregnancy.

Objectives: In this observational study we aimed to evaluate the efficacy and safety of TA in high-risk pregnancies in patients with rheumatic diseases, followed over a decade in a tertiary Center.

Methods: Between January 2005 and April 2019, 843 TA procedures were performed during 51 pregnancies in 43 patients: 745 plasma exchange sessions and 98 immunoabsorption sessions. TA was performed in 29 (57\%) pregnancies of 21 $(48.8 \%)$ patients with antiphospholipid antibody syndrome (APS), in 20 (39.2\%) pregnancies of $20(46.5 \%)$ patients with congenital heart block (CHB), in $1(1.9 \%)$ pregnancy of $1(2.3 \%)$ patient with systemic sclerosis (SSc) and $1(1.9 \%)$ pregnancy of $1(2.3 \%)$ patient affected by lupic nephritis (SLE).

Results: During the period considered, apheresis sessions applied to pregnant women were $7.1 \%$ of the total $(n=13.251)$. The average age at the first treatment was 33 years (range 24-43). The mean management age at the first apheretic treatment was 21 weeks (range 4-32). Twelve (1.4\%) apheresis sessions were complicated by adverse events, none required or prolonged hospitalization. There were $44(86.3 \%)$ live births, $3(5.9 \%)$ spontaneous abortions and 2 (3.9\%) voluntary terminations of pregnancy, $2(3.9 \%)$ lost to follow-up. The average gestational age at birth was 35 weeks (range 24-37) and cesarean section was performed in $41(80.4 \%)$ cases. TA was added to conventional therapy in $24 / 29(82.7 \%)$ patients with APS, to the detection of fetal cardiac activity, while in $5 / 26(17.3 \%)$ it was introduced when the first signs of pregnancy complications such as mild preclampsia, HELLP and IUGR were detected. TA was started within 24 hours of atrioventricular block (AVB) detection; 10/20 (50\%) mothers with $\mathrm{CHB}$ were diagnosed with 2nd degree AVB, $9 / 20$ (45\%) with 3rd degree AVB and one (5\%) with sinus bradycardia and endocardial fibroelastosis. The patient with SSc was treated with TA twice a week from the 32nd SG until delivery, which occurred at the 36th SG, due to severe IUGR and oligohydramnios. The patient with SLE complicated by lupic nephritis was treated with TA twice a week, from the 26th SG until the birth, which took place at the 31st SG

Conclusion: Our data have shown that TA in pregnancy is well tolerated. Close collaboration between rheumatologist, obstetrician and specialist in TA is essential to ensure a successful outcome of high-risk pregnancies.

Disclosure of Interests: : Anna Colpo: None declared, Piero Marson: None declared, Tiziana Tison: None declared, Alessandra Zambon: None declared, Annalisa La Rosa: None declared, Ermella Zanetti: None declared, Amelia Ruffatti: None declared, Giustina De Silvestro: None declared, Ariela Hoxha Speakers bureau: Celgene, UCB, Novartis, Sanofi, Werfen

DOI: 10.1136/annrheumdis-2020-eular.3939

\section{SAT0177 MULTITARGET THERAPY WITH TACROLIMUS AND MYCOPHENOLATE MOFETIL FOR TREATMENT OF LUPUS NEPHRITIS PRESENTED WITH RAPIDLY PROGRESSIVE GLOMERULONEPHRITIS}

Y. Imai ${ }^{1}$, H. Ikeuchi ${ }^{1}$, J. Suwa ${ }^{1}$, Y. Ohishi ${ }^{1}$, M. Watanabe ${ }^{1}$, M. Nakasatomi ${ }^{1}$, H. Hamatani ${ }^{1}$, T. Sakairi ${ }^{1}$, Y. Kaneko ${ }^{1}$, K. Hiromura ${ }^{1}$. ${ }^{1}$ Gunma University Graduate School of Medicine, Department of Nephrology and Rheumatology, Maebashi, Japan

Background: Although, most lupus nephritis patients present with chronic glomerulonephritis or nephrotic syndrome, some patients develop rapidly progressive glomerulonephritis (RPGN), which is a clinical syndrome characterized by rapid loss of renal function over a short period of time (days to months). Multitarget therapy using tacrolimus and mycophenolate mofetil (MMF) has been reported to be effective as induction therapy of Class III to Class V lupus nephritis ${ }^{1}$. However, its efficacy on lupus nephritis presented with RPGN has not been well reported.

Objectives: We aimed to examine the efficacy of multitarget therapy on lupus nephritis presented with RPGN.

Methods: We retrospectively analyzed patients with biopsy-proven lupus nephritis, who clinically showed RPGN, and were treated by multitarget therapy with tacrolimus and MMF in our department. Data were expressed as mean \pm SD.

Results: Five lupus nephritis patients ( 3 female) with RPGN were treated by multitarget therapy as induction therapy. Mean age was $36.6 \pm 13.5$ years old. Renal biopsy at treatment revealed Class IV $(A)$ in 2, Class IV $(A+C)$ in 1 and Class $\mathrm{IV}(\mathrm{A})+\mathrm{V}$ in 2 . The percentage of glomerular crescents was $23.1 \pm 25.4 \%$. eGFR and proteinuria at the initiation of treatment were $46.8 \pm 11.5 \mathrm{~mL} / \mathrm{min} / 1.73 \mathrm{~m}^{2}$ and $7.7 \pm 3.4 \mathrm{~g} / \mathrm{gCr}$, respectively. Patients were initially treated with methylprednisolone pulse therapy followed by $0.8-1.0 \mathrm{mg} / \mathrm{kg}$ of prednisolone (PSL), $2-3 \mathrm{mg} / \mathrm{day}$ of tacrolimus and $1000 \mathrm{mg} /$ day of MMF. At 6 months, eGFR and proteinuria improved to $72.9 \pm 11.3 \mathrm{~mL} / \mathrm{min} / 1.73 \mathrm{~m}^{2}$ and $0.19 \pm 0.13 \mathrm{~g} / \mathrm{gCr}$, respectively. At 12 months, eGFR and proteinuria further improved to $76.8 \pm 7.8 \mathrm{~mL} / \mathrm{min} / 1.73 \mathrm{~m}^{2}$ and $0.10 \pm 0.07 \mathrm{~g} / \mathrm{gCr}$ respectively and the dose of PSL was reduced to $6.6 \pm 1.5 \mathrm{mg} /$ day. Three patients became positive for cytomegalovirus antigenemia and were successfully treated with antiviral therapy.

Conclusion: Multitarget therapy is effective in lupus nephritis even in patients presented with RPGN.

References:

[1] Liu Z, Zhang H, Liu Z, et al. Multitarget therapy for induction treatment of lupus nephritis: a randomized trial. Ann Int Med 2015; 162: 18-26.

Disclosure of Interests: Yoichi Imai: None declared, Hidekazu lkeuchi Speakers bureau: CHUGAI PHARMACEUTICAL CO., LTD.

Astellas Pharma Inc., Junya Suwa: None declared, Yuko Ohishi: None declared Mitsuharu Watanabe: None declared, Masao Nakasatomi: None declared, Hiroko Hamatani: None declared, Toru Sakairi: None declared, Yoriaki Kaneko Grant/ research support from: CHUGAI PHARMACEUTICAL CO., LTD.

Astellas Pharma Inc. b, Speakers bureau: CHUGAI PHARMACEUTICAL CO., LTD. Astellas Pharma Inc., Keiju Hiromura Grant/research support from: CHUGAI PHARMACEUTICAL CO., LTD.

Astellas Pharma Inc., Speakers bureau: CHUGAI PHARMACEUTICAL CO., LTD. Astellas Pharma Inc.

DOI: 10.1136/annrheumdis-2020-eular.1775

\section{SAT0178 $\quad$ HYDROXYCHLOROQUINE CONTROLS DISEASE ACTIVITY IN SLE AND MULTIMODAL IMAGING TECHNIQUES SHOULD BE USED TO DETECT OCULAR TOXICITY}

B. Ince ${ }^{1}$, M. B. Ogurel ${ }^{2}$, Z. Cebeci ${ }^{2}$, Y. B. Tor ${ }^{3}$, B. H. Ucar ${ }^{4}$, Y. Yalçınkaya ${ }^{1}$, A. Gül ${ }^{1}$, M. Inanc ${ }^{1}$, M. L. Ocal ${ }^{1}$, B. Artim-Esen ${ }^{1} .{ }^{1}$ Istanbul University, Istanbul Faculty of Medicine, Division of Rheumatology, Istanbul, Turkey; ${ }^{2}$ Istanbul University, Istanbul Faculty of Medicine, Dept. of Ophthalmology, Istanbul, Turkey; ${ }^{3}$ Istanbul University, Istanbul Faculty of Medicine, Dept. of Internal Medicine, Istanbul, Turkey; ${ }^{4}$ Istanbul University, Istanbul Faculty of Medicine, Istanbul, Turkey

Background: Hydroxychloroquine (HCQ) is an immunomodulatory drug that has been shown to improve disease activity in systemic lupus erythematosus (SLE) However, retinal toxicity is an important concern.

Objectives: In this study we sought to evaluate the effect of $\mathrm{HCQ}$ on disease activity and damage in patients with SLE in whom HCQ was discontinued due to retinal toxicity and whether it could be restarted by a detailed ophthalmologic examination.

Methods: Patients who met SLE SLICC classification criteria and were on HCQ for at least 3 years after reaching Lupus Low Disease Activity State (LLDAS) following remission induction and were followed up for at least 3 years after termination of $\mathrm{HCQ}$ treatment due to retinal toxicity diagnosed with visual field test were analyzed. Disease activity (LLDAS and SLEDAI-2K) and both the number and severity of flares were recorded for each patient whilst on $\mathrm{HCQ}$ and after cessation of treatment. All patients were examined by two experienced ophthalmologists and were assessed by computerized visual field, optical coherence tomography (OCT), fundus autofluorescence (FAF) and fundus florescein angiography (FFA) to further analyze toxicity.

Results: Out of 88 patients with recorded $\mathrm{HCQ}$ retinal toxicity in a cohort of 1500 patients with SLE, 64 patients (mean age at diagnosis $33.4 \pm 10.5(10-57)$ $88.5 \%$ female) with complete data and opthalmologic re-examination results were included in the analyses. The average duration on HCQ was $122 \pm 85$ (39-336) months, and the mean follow-up time was $74.6 \pm 48.3$ (36-239) months after the drug was discontinued. Comparison of mean disease activity in the 3 -year period when patients were on HCQ to 3 years post-cessation revealed a significantly lower mean SLEDAI-2K score in the former $(0.89 \pm 1.28$ vs. $1.3 \pm 1.6, p=0.02)$. The $\%$ of visits maintaining LLDAS was higher during HCQ treatment $(89.7 \pm 17.6$ vs. 80.1 $\pm 23.5, p=0.001$ ). There was significantly a higher frequency of flares with a dominance of mild-moderate types after HCQ was ceased ( 47.5 vs. $26.2 \%, p=0.03 ; 39.3$ vs. $22.9 \%, p=0.024$ respectively). There were more patients with serious flares in the post-discontinuation period but without statistical significance $(13.1 \%$ vs. $4.9 \%$ $\mathrm{p}=0.08)$. Thirty-seven $(60 \%)$ of patients restarted the treatment after ophthalmological examination. Although $38(62,3 \%)$ patients had visual field defects in the latest examination, multimodal imaging with OCT, FAF and FFA revealed that only 19 $(31 \%)$ patient had typical retinal toxicity. Five patients were found to have macula atrophy due to other causes (4 age related macular degeneration and 1 vitromacular adhesion). Since the discrimination of macular pathology would not be possible with imaging in these patients, HCQ was not prescribed. Comparison of patients with and without retinal toxicity showed that duration of $\mathrm{HCQ}$ use and $\mathrm{HCQ}$-free time was not significantly different between patients.

Conclusion: $\mathrm{HCQ}$ is effective in controlling disease activity in patients with SLE and an opportunity for re-medication with $\mathrm{HCQ}$ is valuable. More than half of the 